J Clin Densitom. 2019 ; 22(3): 391-400. doi:10.1016/j.jocd.2018.12.004.

\title{
Statistical models to assess leg muscle mass in ambulatory children with spastic cerebral palsy using dual-energy X-ray absorptiometry
}

\author{
Chuan Zhang ${ }^{1}$, Daniel G. Whitney², Harshvardhan Singh ${ }^{3}$, Jill M. Slade ${ }^{4}$, Ye Shen $^{5}$, \\ Freeman Miller ${ }^{6}$, Christopher M. Modlesky ${ }^{1, *}$ \\ ${ }^{1}$ Department of Kinesiology, University of Georgia, Athens, GA, USA; \\ ${ }^{2}$ Department of Physical Medicine and Rehabilitation, University of Michigan, Ann Arbor, MI, USA; \\ ${ }^{3}$ Department of Physical Therapy, University of Alabama at Birmingham, Birmingham, AL, USA; \\ ${ }^{4}$ Department of Radiology, Michigan State University, East Lansing, MI, USA; \\ ${ }^{5}$ Department of Epidemiology and Biostatistics, University of Georgia, Athens, GA, USA; \\ ${ }^{6}$ Department of Orthopedics, Nemours AI duPont Hospital for Children, Wilmington, DE, USA.
}

\begin{abstract}
Cerebral palsy (CP) is a movement disorder associated with small and weak muscles. Methods that accurately assess muscle mass in children with $\mathrm{CP}$ are scarce. The purpose of this study was to determine whether dual-energy X-ray absorptiometry (DXA) accurately estimates midleg muscle mass in ambulatory children with spastic CP. Ambulatory children with spastic $\mathrm{CP}$ and typically developing children $5-11$ y were studied ( $n=15$ / group). Fat-free soft tissue mass (FFST) and fat mass at the middle third of the tibia (i.e., midleg) were estimated using DXA. Muscle mass (muscle MRI $_{\text {) and muscle mass corrected for intramuscular fat (muscle }}$ MRIfc) in the midleg were estimated using magnetic resonance imaging (MRI). Statistical models were created to predict muscle MRI $_{\text {and muscle }}$ MRIfc using DXA. Children with CP compared to typically developing children had lower FFST (38\%), muscle $_{\mathrm{MRI}}(40 \%)$ and muscle $\mathrm{MRIfc}_{\mathrm{c}}(47 \%)$ (all $p<$

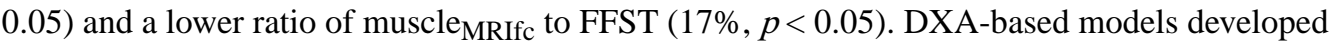
using data from typically developing children overestimated muscle ${ }_{\mathrm{MRI}}(13 \%)$ and muscle MRIfc $_{\text {. }}$ (22\%) (both $p<0.05$ ) in children with CP. DXA-based models developed using data from children with CP explained $91 \%$ of the variance in muscle ${ }_{\mathrm{MRI}}$ and $90 \%$ of the variance in muscle $\mathrm{MRIfc}_{\mathrm{in}}$ in children with CP (both $p<0.05$ ). Moreover, the estimates were not different from muscle MRI $_{\text {and }}$ muscle $_{\text {MRIfc }}$ (both $p>0.99$ ). We conclude that DXA-based statistical models accurately estimate midleg muscle mass in children with $\mathrm{CP}$ when the models are composed using data from children with CP rather than typically developing children.
\end{abstract}

\footnotetext{
*Address correspondence to: Christopher M. Modlesky, PhD, Department of Kinesiology, University of Georgia, 330 River Road, Room 353, Athens, GA, 30602, Telephone: 706-542-4424, Fax: 706-542-3417, christopher.modlesky@uga.edu.

Publisher's Disclaimer: This is a PDF file of an unedited manuscript that has been accepted for publication. As a service to our customers we are providing this early version of the manuscript. The manuscript will undergo copyediting, typesetting, and review of the resulting proof before it is published in its final citable form. Please note that during the production process errors may be discovered which could affect the content, and all legal disclaimers that apply to the journal pertain.
} 


\section{Keywords}

muscle mass; cerebral palsy; dual-energy X-ray absorptiometry; magnetic resonance imaging; intramuscular fat

\section{INTRODUCTION}

As the largest tissue component of the lean body mass in humans, skeletal muscle plays an important role in daily life. Apart from force-generating capacity (1), which is crucial for movement and balance (2), it is also involved in numerous physiological processes that help the body achieve homeostasis (3). Altered skeletal muscle quantity and quality have been associated with multiple morbidities, such as insulin resistance (4), functional impairment and disability (5), obesity (6) and osteoporosis (7). Therefore, obtaining information about human skeletal muscle development and accretion is necessary for disease prevention and treatment.

Magnetic resonance imaging (MRI) is considered the "gold standard" for noninvasive skeletal muscle mass assessment (8). However, some limitations associated with MRI include high cost, low availability, and time-consuming acquisition and processing procedures. An alternative to MRI is dual-energy X-ray absorptiometry (DXA) because of its lower cost, and shorter scanning and processing time. Studies have shown that fat-free soft tissue (FFST) from DXA can be used to estimate skeletal muscle mass (9-12). However, few studies have focused on children $(9,10,12)$ and fewer have focused on children with arrested muscle accretion, such as children with cerebral palsy (CP) (9). Muscle assessment in children with $\mathrm{CP}$ is particularly important because $\mathrm{CP}$ is the most common childhood disability and it is associated with muscles that are small (13-15), weak (15) and highly infiltrated with fat (16). The muscle size deficit in children with CP is profound in the lower extremities, especially the legs (13). Moreover, the concentration of intramuscular fat in the legs is $~ 50 \%$ higher in ambulatory children with CP than in typically developing children (16).

There is evidence that a DXA-based statistical model developed for typically developing children overestimates skeletal muscle mass in nonambulatory children with $\mathrm{CP}$, which has been linked to their lower proportion of muscle in the FFST (9). Whether a similar problem is present and correctable in ambulatory children with spastic CP is unknown. In addition, most MRI structural images used to assess muscle volume (MV) cannot separate intramuscular fat from muscle tissue. Therefore, the proportion of muscle in the FFST may be even lower and the overestimation of muscle mass by DXA-based models higher in children with $\mathrm{CP}$ than previously demonstrated when the high degree of intramuscular fat is considered.

The objective of this study was to determine whether DXA can be used to estimate midleg muscle mass in ambulatory children with spastic CP. It was hypothesized that midleg muscle mass in children with CP would be overestimated by a DXA-based statistical model developed using data from typically developing children while a model developed using data from children with $\mathrm{CP}$ would yield accurate estimation. 


\section{METHODS}

\section{Participants}

Sixteen ambulatory children with spastic $\mathrm{CP}(\mathrm{n}=5$ girls; $\mathrm{n}=12$ Caucasian, $\mathrm{n}=2$ African American and $\mathrm{n}=2$ Hispanic) and between 5 and 11 years old were recruited from local pediatric hospitals and clinics. Fifteen typically developing children $(n=4$ girls; $n=11$ Caucasian, $\mathrm{n}=2$ African American, $\mathrm{n}=1$ Hispanic and $\mathrm{n}=1$ Asian) without known neurological disorders and had similar ages to children with $\mathrm{CP}$ also participated. This study received approval from the Institutional Review Board. Written consent and assent were obtained from the participant and the parent, respectively.

\section{Anthropometrics}

Height was assessed using a stadiometer (Seca 217; Seca GmbH \& Co. KG., Hamburg, GER). Body mass was assessed using a digital weight scale (Detecto 6550, Cardinal Scale, Webb City, MO). Both measurements were taken while children wore minimal clothing. Body mass index (BMI) was subsequently calculated. Height, body mass and BMI percentiles were determined using growth charts (17).

\section{Sexual Maturity}

Sexual maturity was assessed using the Tanner staging technique (18). This is a five-point scale with I indicating no development and $\mathrm{V}$ indicating full development. There are two parts to this assessment. Pubic hair development in both boys and girls, and testicular/penile development in boys and breast development in girls were assessed by a physician assistant.

\section{Gross Motor Function}

Gross motor function in children with $\mathrm{CP}$ was classified according to the gross motor function classification system (GMFCS) (19). GMFCS is a five-point scale ranging from I to $\mathrm{V}$ with a higher number indicating lower gross motor function. Participants with $\mathrm{CP}$ in this study had GMFCS I or II.

\section{DXA}

FFST and fat mass at the level of the middle-third (i.e., midleg) of the nondominant side in typically developing children and the more affected side in children with $\mathrm{CP}$ were assessed using DXA (Delphi W, version 11.2; Whole Body Analysis; Hologic Inc., Marlborough, MA) and a whole body scan. The BodyFIX (Medical Intelligence, Inc., Schwabmúnchen, GER) was used to limit motion in children with CP (9). After completion of the scan, a region of interest (ROI) box was placed at the middle-third of the tibia to represent the midleg and fat mass and FFST were determined. The coefficient of variation (CV) for repeat measures of FFST and fat mass in the midleg were $0.6 \%$ and $0.7 \%$, respectively (9).

MRI

Midleg muscle mass and muscle mass corrected for intramuscular fat were determined using MRI (GE, 1.5 T, Waukesha, WI) in the same leg tested using DXA. To help children remain still during the MRI testing, they were secured from the waist down using the BodyFix to 
limit motion (16). They also watched a movie of their choice using an MRI-compatible goggle system (CinemaVision, Resonance Technology Inc, Northridge, CA). All scans with visible motion in the images were redone. Axial images $(0.5 \mathrm{~cm}$ slice thickness and $0.5 \mathrm{~cm}$ spacing) were collected from the tibia plateau to the malleolar surface using a semiflex long bone array coil (ScanMed, Omaha, NE) and two scans. The first scan (TR $=650, \mathrm{TE}=14$, $\mathrm{NEX}=3$, Bandwidth $=15.63$, frequency 512 and phase 256, field of view $12 \mathrm{~cm}$ ) yielded T1-weighted images. The second scan (IDEAL: fast-spin-echo, $\mathrm{TR}=600, \mathrm{TE}=\min$ full, $\mathrm{NEX}=2$, Bandwidth $=31.25$, frequency 320 and phase 224) yielded fat and water images.

Images were processed with a program developed using Interactive Data Language (Research Systems, Inc., Boulder, CO). T1-weighted images at the level of the midleg were automatically identified and processed. Skeletal muscle area was separated from other tissues (20) with minor manual adjustments applied as needed. The identified area for the first and last images were multiplied by a correction factor $(<1)$, and the rest of the images were multiplied by 1 to account for the slice thickness and spacing, and sum of all results was defined as mid-third tibia MV. The mid-third tibia MV was then multiplied by 1.04 $\mathrm{g} / \mathrm{cm}^{3}$, the assumed density of muscle (21) to calculate muscle mass (muscle MRI $_{\text {). The }}$ reliability in our laboratory is excellent as indicated by intraclass correlation coefficients > 0.99 and CVs of $0.5 \%$ (9).

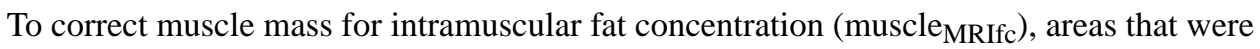
assigned to muscle in the T1-weighted images were used to identify muscle areas in the corresponding fat and water images. The signal intensity (SI) was used to calculate fat concentration using the following equation: Fat concentration $=$ SI from fat images $/$ (SI from fat images + SI from water images) $* 100$ (22). Skeletal MV corrected for intramuscular fat concentration was calculated using the following equation: Corrected MV $=\mathrm{MV}-(\mathrm{MV} \mathrm{x}$ fat concentration). The corrected MV was then multiplied by the assumed muscle density (21) to yield muscle MRIfc.

\section{Statistical Analysis}

Data analyses were conducted using SPSS (version 24.0; SPSS, Chicago, IL). Physical characteristics and data from DXA and MRI were checked for normality first, and group comparisons were made using independent t-tests or Mann-Whitney test accordingly. The magnitude of the effects was determined using Cohen's $d(d)$, with $0.2,0.5$ and 0.8 indicating small, medium, and large effects, respectively (23).

A simple linear regression analysis was performed using FFST as the independent variable

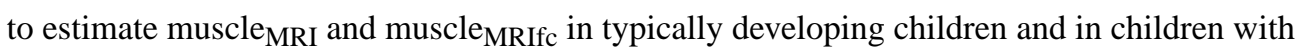
CP. Age, height, body mass, BMI and fat mass were added to the model as predictors one at a time to determine whether those variables could significantly improve model predictability. The final models were cross-validated using the leave-one-out technique (24). Paired t-tests were performed to determine whether model estimated muscle mass was different from muscle $_{\text {MRI }}$ and muscle ${ }_{\text {MRIfc. }}$. 


\section{RESULTS}

One Caucasian boy with CP did not complete the second MRI scan (IDEAL) and was excluded from the analyses. Physical characteristics for the remaining participants are summarized in Table 1. Eight children with CP had GMFCS I and 7 had GMFCS II. No group differences in age, body mass, BMI, BMI percentile, sexual maturity, or fat mass were detected (all $p>0.05$ ). Children with $\mathrm{CP}$ had a lower body mass percentile, height, and height percentile (all $p<0.05$ ). Children with CP also had 38\% lower FFST, $40 \%$ lower

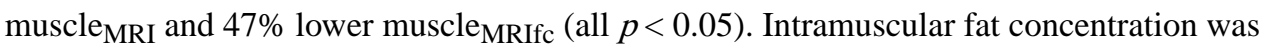
higher in children with CP (24\% vs. $15 \% ; p=0.001)$. Children with CP had a $17 \%$ lower ratio of muscle $_{\text {MRIFC }}$ to FFST ( $0.62 \pm 0.13$ vs. $0.75 \pm 0.06 ; d=1.205, p=0.004$; Fig. $\left.1 \mathrm{~B}\right)$. Although children with $\mathrm{CP}$ also had a lower muscle $\mathrm{MRI}_{\mathrm{I}}$ to FFST ratio, the difference was not statistically significant $(0.82 \pm 0.13$ vs. $0.88 \pm 0.06 ; d=0.564, p=0.138$; Fig. $1 \mathrm{~A})$. A visual depiction of the muscle and fat discrepancies between a boy with $\mathrm{CP}$ and an age-matched typically developing boy is shown in Fig 2 .

\section{Statistical model developed using typically developing children}

Simple linear regression using FFST as the predictor explained $95 \%$ of the variance in muscle $_{\text {MRI }}$ in typically developing children (Table 2, model 1). Adding age or height didn't significantly improve the model (both $p>0.05$ ). However, adding body mass, BMI or fat mass individually significantly improved the model (all $p<0.05$ ). Adding fat mass yielded

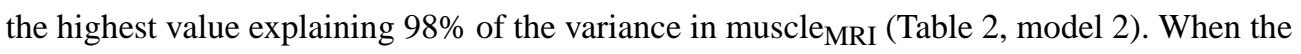
model with FFST and fat mass was cross-validated using the leave-one-out method, the estimated muscle mass (muscle DXA $_{\text {}}$ ) was strongly related $\left(r^{2}=0.97\right.$, SEE $=11.8 \mathrm{~g}, p<$ $0.001)$ and not different from muscle $\mathrm{MRI}(p=0.770)$. When the model was cross-validated in children with $\mathrm{CP}$, muscle $\mathrm{DXA}_{\mathrm{DA}}$ was strongly related to muscle $\mathrm{MRI}_{\mathrm{I}}$, as shown in the scatter plot in Fig. 3A $\left(r^{2}=0.90, \mathrm{SEE}=20.0 \mathrm{~g}, p<0.001\right)$. However, the model overestimated muscle $_{\text {MRI }}$ by $13 \%(17.3 \mathrm{~g}, p=0.004)$, as shown by most data points and the regression line residing mainly below the line of identity in Fig. $3 \mathrm{~A}$ and by most data points and mean difference line above the no difference line in the Bland-Altman plot in Fig. 3B.

Simple linear regression using FFST as the predictor explained $96 \%$ of the variance in muscle $_{\text {MRIfc }}$ in typically developing children (Table 3, model 1). None of the additional predictors significantly improved the model (all $p>0.05$ ). When the final model with only FFST as the predictor was cross-validated using the leave-one-out method, the estimated muscle mass was also strongly related $\left(r^{2}=0.95\right.$, SEE $\left.=12.7 \mathrm{~g}, p<0.001\right)$ and not different from muscle MRIfc $(p=0.858)$. When the same model was cross-validated in children with $\mathrm{CP}$, the estimated muscle mass (muscle ${ }_{\mathrm{DXAfc}}$ ) was strongly related to muscle ${ }_{\mathrm{MRIfc}}$, as shown in Fig. 4A $\left(r^{2}=0.83\right.$, SEE $\left.=17.6 \mathrm{~g}, p<0.001\right)$. However, the model overestimated muscle $_{\text {MRIfc }}$ by $22 \%$ (22.3 g, $p=0.001$ ), as shown by most data points and the regression line residing below the line of identity in Fig. $4 \mathrm{~A}$ and by most data points and the mean difference line above the no difference line in the Bland-Altman plot in Fig. 4B.

The difference between muscle DXA $_{\text {and muscle }}$ MRI in children with $\mathrm{CP}$ was inversely related to the ratio of muscle mass to FFST $(r=-0.79, p<0.001)$, as shown in Fig. 5A. The positive values for the difference between muscle $_{\mathrm{DXA}}$ and muscle $\mathrm{e}_{\mathrm{MRI}}$ indicate an 
overestimation of muscle mass by the DXA-based model, which was greatest in children

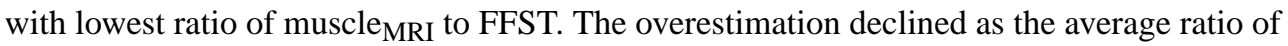
muscle to FFST in typically developing children (0.88) was approached. A similar inverse relationship was demonstrated between the difference in predicted muscle mass and

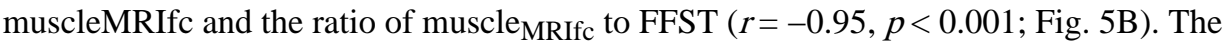
overestimation was greatest in children with lowest ratio of muscle to FFST and became smaller as the average ratio of muscle to FFST in typically developing children (0.75) was approached.

\section{Statistical model developed using children with CP}

A simple regression model using FFST as the predictor explained $91 \%$ of the variance in muscle $_{\text {MRI }}$ in children with CP (Table 2, model 3). Adding age, height, body mass, BMI or fat mass as independent variables didn't significantly improve the model (all $p>0.05$ ). Estimated muscle mass using the model with FFST as the predictor was not different from muscle $_{\text {MRI }}(p>0.990)$. The validity of estimation was excellent as shown by most data points and the regression line residing near the line of identity in Fig. $6 \mathrm{~A}$ and by most data points and the mean difference line near the no difference line in the Bland-Altman plot in Fig. 6B. When the model was cross-validated using the leave-one out method, the estimated muscle mass was strongly related $\left(r^{2}=0.87 \mathrm{SEE}=21.8 \mathrm{~g}, p<0.001\right)$ and not different from muscle $_{\text {MRI }}(p=0.893)$.

A second regression model using FFST as the predictor explained $83 \%$ of the variance in muscle $_{\text {MRIfc }}$ in children with CP (Table 3, model 2). Adding age, height, BMI or fat mass didn't significantly improve the model (all $p>0.05$ ). However, when body mass was added to the model, significant improvement was observed with $90 \%$ of the variance in muscle $_{\text {MRIfc }}$ explained (Table 3, model 3). Estimated corrected muscle mass using this model was not different from muscle MRIfc $(p=0.990)$. The validity of estimation was excellent as shown by most data points and the regression line residing near the line of identity in Fig. 7A and by most data points and the mean difference line near the nodifference line in the Bland-Altman plot in Fig. 7B. When the model was cross-validated using the leave-one out method, the estimated corrected muscle mass was strongly related $\left(r^{2}=0.83, \mathrm{SEE}=20.5 \mathrm{~g}, p<0.001\right)$ and not different from muscle ${ }_{\text {MRIfc }}(p=0.785)$.

\section{DISCUSSION}

To our knowledge, this is the first study to show that a DXA-based statistical model can provide accurate estimates of leg muscle mass in ambulatory children with spastic CP. Although models produced using typically developing children provided accurate estimates of muscle mass in typically developing children, they overestimated muscle mass by $13 \%$ and $22 \%$, respectively, before and after correction for intramuscular fat in children with CP. The findings are important because children with CP have very low muscle mass in the leg and methods that provide accurate estimates of muscle mass are needed.

The finding that statistical models developed for typically developing children do not provide accurate estimates of muscle mass in children with $\mathrm{CP}$ is consistent with a previous study. In a small group of nonambulatory children with $\mathrm{CP}$, midthigh muscle mass was 
overestimated by $12-15 \%(p<0.05)$ when a DXA-based model developed using typically developing children was used. The overestimation was attributed to the lower ratio of muscle to FFST in children with CP. An inverse relationship between this ratio and the degree of muscle mass overestimation supports the strong tie between the relative amount of muscle in the FFST and the accuracy of muscle mass estimated by DXA (9). Similarly, in the present study, muscle mass was overestimated by $13 \%$ when a model developed using typically developing children was employed, and the ratio of muscle to FFST was lower in the children with CP. Moreover, there was a strong inverse relationship between this ratio and the degree of muscle mass overestimation by a DXA-based models produced for typically developing children. Other than skeletal muscle, FFST also contains skin, vessels and other connective tissues. Assuming the nonskeletal muscle portion of FFST is not altered in children with $\mathrm{CP}$, the compromised muscle mass will lead to a lower ratio of muscle ${ }_{\mathrm{MRI}}$ to

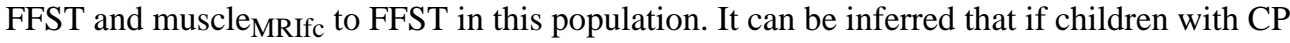
had similar ratios of muscle mass to FFST as typically developing children, there would be no, or minimal overestimation, as indicated in Fig. 5.

Interestingly, in typically developing children, when fat mass was included as a predictor with FFST, the model improved significantly. However, the same improvement was not found in children with CP. Although both groups had a similar amount of fat mass as measured by DXA, the fat mass in children with $\mathrm{CP}$ actually represented a larger proportion of the leg soft tissue, as children with CP had 38\% smaller FFST. Similarly, a study that assessed thigh skeletal muscle mass in men with spinal cord injury found a stronger relation between the difference in skeletal muscle and FFST when adipose tissue was accounted for in controls than that in people with spinal cord injury (25). The reason fat mass contributes to DXA-derived estimates of skeletal muscle mass in a healthy population but not in clinical populations who manifest reduced muscle mass is currently unknown but warrants further investigation.

To our knowledge, this is the first study to assess the accuracy of DXA estimates of muscle mass adjusted for intramuscular fat. This is important because intramuscular fat does not contribute to the force production of muscle and has been shown to be associated with decreased physical function (26). Compared to typically developing children, children with $\mathrm{CP}$ have a higher intramuscular fat concentration. Therefore, the disparity in muscle mass was even greater when intramuscular fat was subtracted from muscle mass (47\% vs. $40 \%$ lower muscle mass in children with $\mathrm{CP}$ ). As a result, DXA overestimated muscle mass corrected for fat concentration to a greater degree (22\% vs. $13 \%$, respectively). A DXAbased model developed for children with CP yielded much better accuracy, as indicated by the high amount of variance (90\%) explained by the model and no difference in DXA and MRI estimates of muscle mass.

Small muscles have been linked to poor physical function and low participation in physical activity $(14,27)$, and thus may increase risk of chronic disease $(28,29)$. The risk is likely to rise as individuals with $\mathrm{CP}$ age $(30,31)$. This idea is supported by the much higher cardiovascular (32) and osteoporosis (31) risks in adults with CP compared to general population. Therefore, the muscle deficit in children with $\mathrm{CP}$ is an important therapeutic target for interventions aimed at improving physical function and physical activity and 
reducing long-term risk of developing chronic diseases. The present study ascertains the validity of using DXA to predict leg muscle mass, a region that has been found to have the largest muscle deficit in individuals with CP (13). The findings suggest that the DXA-based models developed in the present study provide an accessible, relatively cheap and accurate method to assess the skeletal muscle status in ambulatory children with spastic CP. However, studies are needed to determine whether the models can accurately capture the muscle changes in children with $\mathrm{CP}$ due to natural growth or treatment, such as resistance training (33), vibration treatment (34), and botulinum toxin (35).

There are limitations associated with this study. First, because of the relatively small number of subjects in each group, the results should be interpreted with caution. However, no differences in age or sexual maturity were observed and the composition of boys and girls was similar. Second, to estimate skeletal muscle mass, muscle volume was multiplied by $1.04 \mathrm{~g} / \mathrm{cm}^{3}$, which is the assumed muscle density of muscle determined from adult cadavers (21). It's possible that for children that are still growing, their true muscle density may not be the same as adults. It is also reasonable to infer that for children with $\mathrm{CP}$ whose muscle volume and quality are compromised, their muscle density may be lower than their typically developing peers. Therefore, the true muscle deficit in children with CP may be at a greater magnitude than what was presented in the current study. However, this potential discrepancy has been partly mediated by correcting for intramuscular fat concentration. The assumed density of fat (36) is lower than muscle, so the density of muscle for children with CP after correcting for fat concentration would be closer to that of typically developing children. Third, the findings from the present study can't be extrapolated to other regions of the body.

In conclusion, DXA-based statistical models can accurately estimate midleg muscle mass in ambulatory children with spastic $\mathrm{CP}$ when the models are developed using data from children with CP. Models developed using data from typically developing children overestimate leg muscle mass in children with $\mathrm{CP}$ due to the lower proportion of muscle in the FFST of children with CP. Future studies are needed to determine whether the models developed in the present study are valid in children with more severe forms of $\mathrm{CP}$ and whether the models accurately estimate change that occurs with growth or intervention. Future studies are also needed to develop models to estimate muscle mass at other body regions in children with $\mathrm{CP}$.

\section{Supplementary Material}

Refer to Web version on PubMed Central for supplementary material.

\section{ACKNOWLEDGEMENTS}

This work was supported by the National Institutes of Health, grant numbers HD071397 and HD090126.

\section{REFERENCES:}

1. Sandow A Fundamental mechanics of skeletal muscle contraction. Am J Phys Med. 1952;31(2):103-25. [PubMed: 14933587] 
2. Szulc P, Beck TJ, Marchand F, and Delmas PD. Low skeletal muscle mass is associated with poor structural parameters of bone and impaired balance in elderly men--the MINOS study. J Bone Miner Res. 2005;20(5):721-9. [PubMed: 15824844]

3. Pedersen BK, and Febbraio MA. Muscles, exercise and obesity: skeletal muscle as a secretory organ. Nat Rev Endocrinol. 2012;8(8):457-65. [PubMed: 22473333]

4. Albu JB, Kovera AJ, Allen L, Wainwright M, Berk E, Raja-Khan N, et al. Independent association of insulin resistance with larger amounts of intermuscular adipose tissue and a greater acute insulin response to glucose in African American than in white nondiabetic women. Am J Clin Nutr. 2005;82(6):1210-7. [PubMed: 16332653]

5. Janssen I, Heymsfield SB, and Ross R. Low relative skeletal muscle mass (sarcopenia) in older persons is associated with functional impairment and physical disability. J Am Geriatr Soc. 2002;50(5):889-96. [PubMed: 12028177]

6. Sinha R, Dufour S, Petersen KF, LeBon V, Enoksson S, Ma YZ, et al. Assessment of skeletal muscle triglyceride content by (1)H nuclear magnetic resonance spectroscopy in lean and obese adolescents: relationships to insulin sensitivity, total body fat, and central adiposity. Diabetes. 2002;51(4):1022-7. [PubMed: 11916921]

7. Kaji H Linkage between muscle and bone: common catabolic signals resulting in osteoporosis and sarcopenia. Curr Opin Clin Nutr Metab Care. 2013;16(3):272-7. [PubMed: 23481148]

8. Mitsiopoulos N, Baumgartner RN, Heymsfield SB, Lyons W, Gallagher D, and Ross R. Cadaver validation of skeletal muscle measurement by magnetic resonance imaging and computerized tomography. J Appl Physiol (1985). 1998;85(1):115-22. [PubMed: 9655763]

9. Modlesky CM, Cavaiola ML, Smith JJ, Rowe DA, Johnson DL, and Miller F. A DXA-based mathematical model predicts midthigh muscle mass from magnetic resonance imaging in typically developing children but not in those with quadriplegic cerebral palsy. J Nutr. 2010;140(12):2260-5 . [PubMed: 20980659]

10. Kim J, Shen W, Gallagher D, Jones A Jr., Wang Z, Wang J, et al. Total-body skeletal muscle mass: estimation by dual-energy X-ray absorptiometry in children and adolescents. Am J Clin Nutr. 2006;84(5):1014-20. [PubMed: 17093152]

11. Kim J, Wang Z, Heymsfield SB, Baumgartner RN, and Gallagher D. Total-body skeletal muscle mass: estimation by a new dual-energy X-ray absorptiometry method. Am J Clin Nutr. 2002;76(2):378-83. [PubMed: 12145010]

12. Midorikawa T, Ohta M, Hikihara Y, Torii S, and Sakamoto S. Predicting skeletal muscle mass from dual-energy X-ray absorptiometry in Japanese prepubertal children. Eur J Clin Nutr. 2017;71(10):1218-22. [PubMed: 28378848]

13. Lampe R, Grassl S, Mitternacht J, Gerdesmeyer L, and Gradinger R. MRT-measurements of muscle volumes of the lower extremities of youths with spastic hemiplegia caused by cerebral palsy. Brain Dev. 2006;28(8):500-6. [PubMed: 16690238]

14. Johnson DL, Miller F, Subramanian P, and Modlesky CM. Adipose tissue infiltration of skeletal muscle in children with cerebral palsy. J Pediatr. 2009;154(5):715-20. [PubMed: 19111321]

15. Elder GCB, Kirk J, Stewart G, Cook K, Weir D, Marshall A, et al. Contributing factors to muscle weakness in children with cerebral palsy. Dev Med Child Neurol. 2003;45(8):542-50. [PubMed: 12882533]

16. Whitney DG, Singh H, Miller F, Barbe MF, Slade JM, Pohlig RT, et al. Cortical bone deficit and fat infiltration of bone marrow and skeletal muscle in ambulatory children with mild spastic cerebral palsy. Bone. 2017;94:90-7. [PubMed: 27732905]

17. Kuczmarski RJ, Ogden CL, Grummer-Strawn LM, Flegal KM, Guo SS, Wei R, et al. CDC growth charts: United States. Adv Data. 2000;314(314):1-27.

18. Tanner J Growth and Adolescence. Oxford: Blackwell Scientific Publications; 1962.

19. Wood E, and Rosenbaum P. The gross motor function classification system for cerebral palsy: a study of reliability and stability over time. Dev Med Child Neurol. 2000;42(5):292-6. [PubMed: 10855648]

20. Suckling J, Sigmundsson T, Greenwood K, and Bullmore ET. A modified fuzzy clustering algorithm for operator independent brain tissue classification of dual echo MR images. Magn Reson Imaging. 1999;17(7):1065-76. [PubMed: 10463658] 
21. Snyder WS, Cook MJ, Nasset ES, Karhauserr LR, Howells GP, and Tipton IH. International Comission on Radiological Protection. Oxford; 1975.

22. Slade JM, Coe LM, Meyer RA, and McCabe LR. Human bone marrow adiposity is linked with serum lipid levels not T1-diabetes. J Diabetes Complications. 2012;26(1):1-9. [PubMed: 22257906]

23. Cohen J Statistical Power for the Behavioral Sciences. Hillsdale, NJ: Lawrence Erlbaum Associates; 1988.

24. Hawkins DM, Basak SC, and Mills D. Assessing model fit by cross-validation. J Chem Inf Comput Sci. 2003;43(2):579-86. [PubMed: 12653524]

25. Modlesky CM, Bickel CS, Slade JM, Meyer RA, Cureton KJ, and Dudley GA. Assessment of skeletal muscle mass in men with spinal cord injury using dual-energy X-ray absorptiometry and magnetic resonance imaging. J Appl Physiol (1985). 2004;96(2):561-5. [PubMed: 14527962]

26. Therkelsen KE, Pedley A, Hoffmann U, Fox CS, and Murabito JM. Intramuscular fat and physical performance at the Framingham Heart Study. Age. 2016;38(2).

27. Visser M, Goodpaster BH, Kritchevsky SB, Newman AB, Nevitt M, Rubin SM, et al. Muscle mass, muscle strength, and muscle fat infiltration as predictors of incident mobility limitations in wellfunctioning older persons. J Gerontol A Biol Sci Med Sci. 2005;60(3):324-33. [PubMed: 15860469]

28. Visser M, Pahor M, Taaffe DR, Goodpaster BH, Simonsick EM, Newman AB, et al. Relationship of interleukin- 6 and tumor necrosis factor-alpha with muscle mass and muscle strength in elderly men and women: The health ABC study. J Gerontol A Biol Sci Med Sci. 2002;57(5):M326-M32. [PubMed: 11983728]

29. Kim S, Won CW, Kim BS, Choi HR, and Moon MY. The Association between the Low Muscle Mass and Osteoporosis in Elderly Korean People. J Korean Med Sci. 2014;29(7):995-1000. [PubMed: 25045234]

30. Peterson MD, Gordon PM, Hurvitz EA, and Burant CF. Secondary muscle pathology and metabolic dysregulation in adults with cerebral palsy. Am J Physiol Endocrinol Metab. 2012;303(9):E1085-93. [PubMed: 22912367]

31. Whitney DG, Hurvitz EA, Devlin MJ, Caird MS, French ZP, Ellenberg EC, et al. Age trajectories of musculoskeletal morbidities in adults with cerebral palsy. Bone. 2018;114:285-91. [PubMed: 29981509]

32. Whitney DG, Hurvitz EA, Ryan JM, Devlin MJ, Caird MS, French ZP, et al. Noncommunicable disease and multimorbidity in young adults with cerebral palsy. Clin Epidemiol. 2018;10:511-9. [PubMed: 29750055]

33. McNee AE, Gough M, Morrissey MC, and Shortland AP. Increases in muscle volume after plantarflexor strength training in children with spastic cerebral palsy. Dev Med Child Neurol. 2009;51(6):429-35. [PubMed: 19170722]

34. Gusso S, Munns CF, Colle P, Derraik JGB, Biggs JB, Cutfield WS, et al. Effects of whole-body vibration training on physical function, bone and muscle mass in adolescents and young adults with cerebral palsy. Sci Rep-Uk. 2016;22518.

35. Williams SA, Reid S, Elliott C, Shipman P, and Valentine J. Muscle volume alterations in spastic muscles immediately following botulinum toxin type-A treatment in children with cerebral palsy. Dev Med Child Neurol. 2013;55(9):813-20. [PubMed: 23789782]

36. Fidanza F, Keys A, and Anderson JT. Density of body fat in man and other mammals. J Appl Physiol. 1953;6(4):252-6. [PubMed: 13108819] 


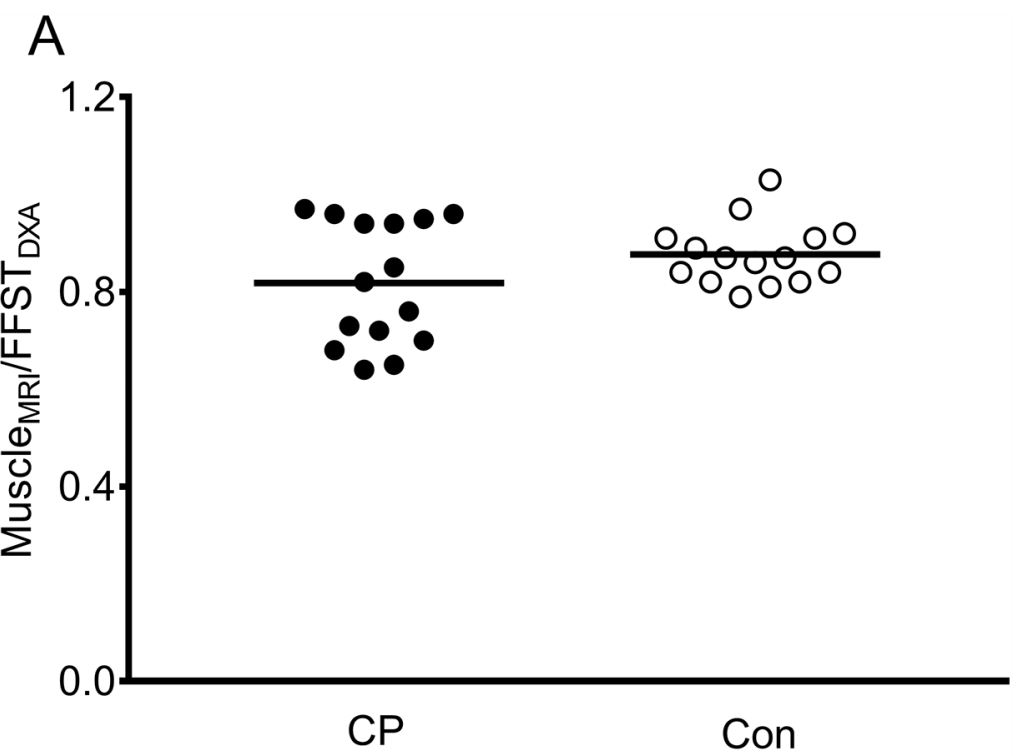

B

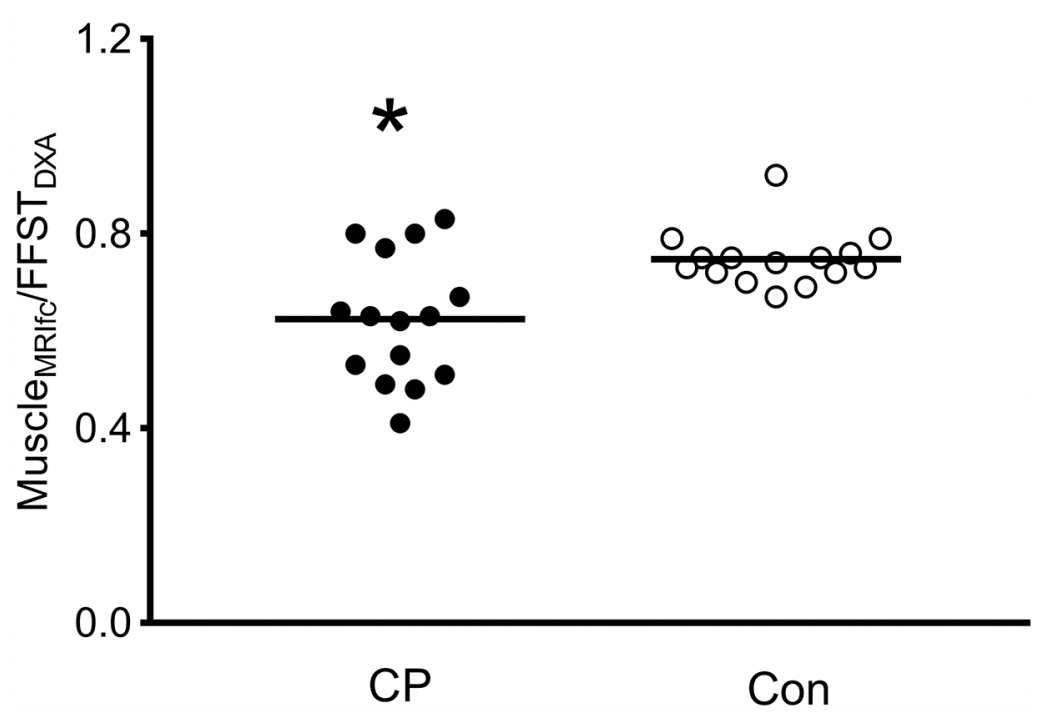

Fig. 1.

Ratio of muscle mass from MRI (Muscle ${ }_{\text {MRI }}$ ) to fat-free soft tissue from DXA (FFST DXA; $_{\text {; }}$ A) and muscle mass from MRI corrected for fat concentration (Muscle MRIfc $_{\text {) }}$ to FFST $_{\text {DXA }}$ (B) in the midleg of children with cerebral palsy $(\mathrm{CP} ; \mathrm{n}=15)$ and typically developing children (Con; $\mathrm{n}=15)$. *Group difference, $p<0.05$. 


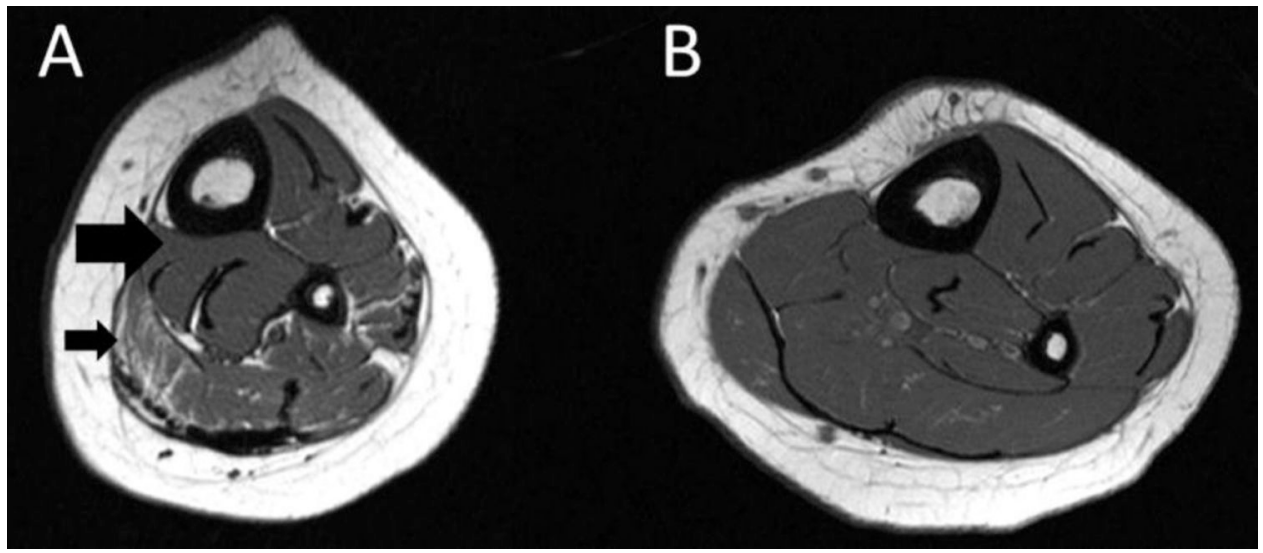

Fig. 2.

Magnetic resonance images from a boy with cerebral palsy (CP; A) and a typically developing boy (B) both 8.5 years of age at the level of the midtibia. The boy with CP had much lower muscle mass (large black arrow in A) and much higher intramuscular fat (smaller black arrow in A) compared to his typically developing peer. 

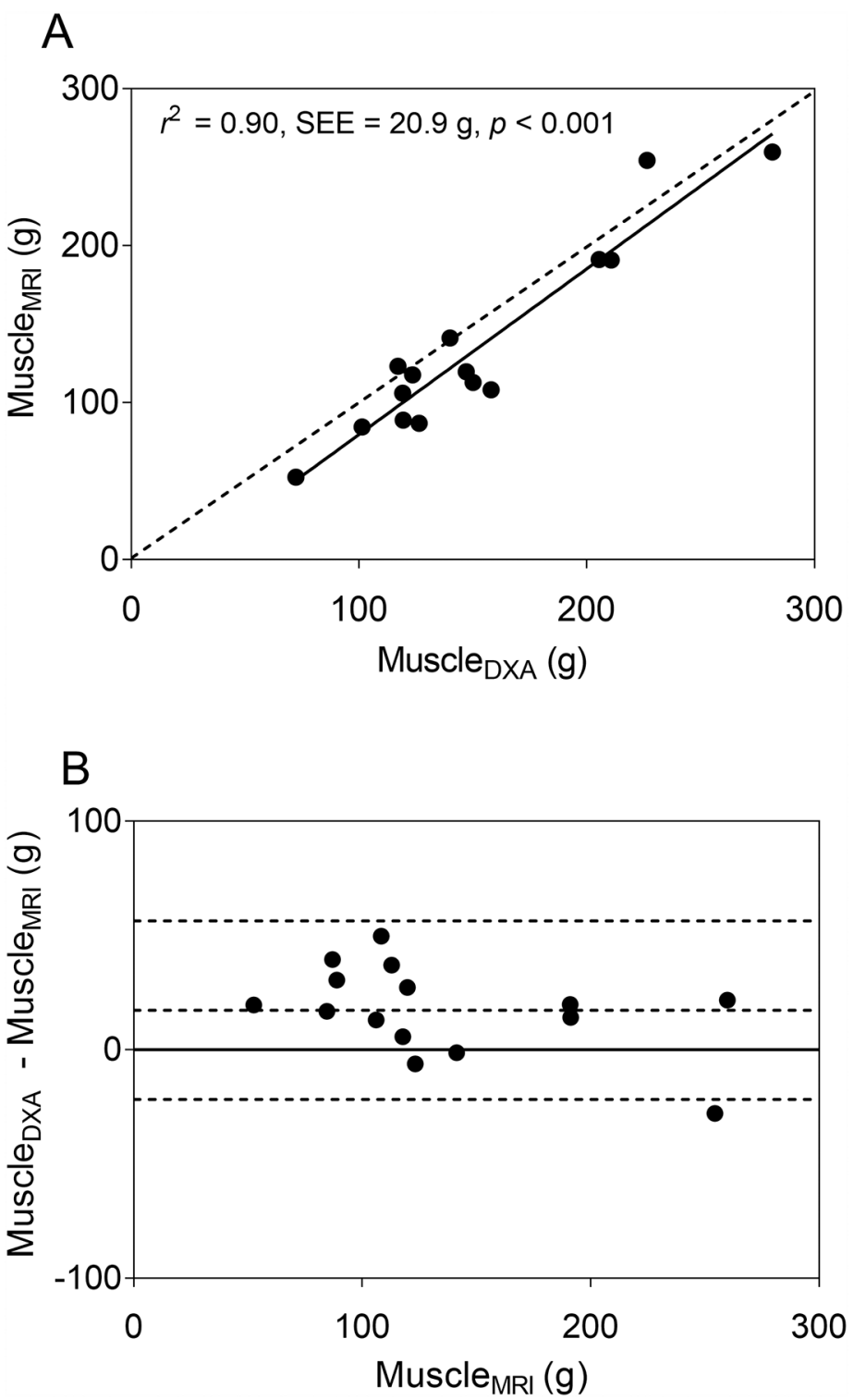

Fig. 3.

(A) The scatter plot shows midleg muscle mass (Muscle $\left.{ }_{M R I}\right)$ in children with cerebral palsy $(\mathrm{CP} ; \mathrm{n}=15$ ) estimated using a dual-energy X-ray absorptiometry (DXA) -based model (Muscle DXA $_{\text {DX }}$ ) developed using data from typically developing children. Muscle DXA was estimated using fat-free soft tissue mass (FFST) from DXA. The dotted line represents the line of identity. The solid line represents the regression line. (B) The Bland-Altman plot

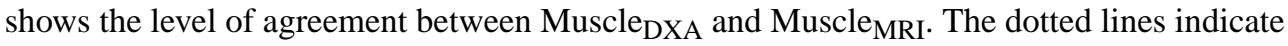
the mean difference \pm 2 SD between actual muscle mass from MRI and the estimated muscle mass by the DXA-based model in children with CP. The solid line indicates no difference between actual and estimated muscle mass. 


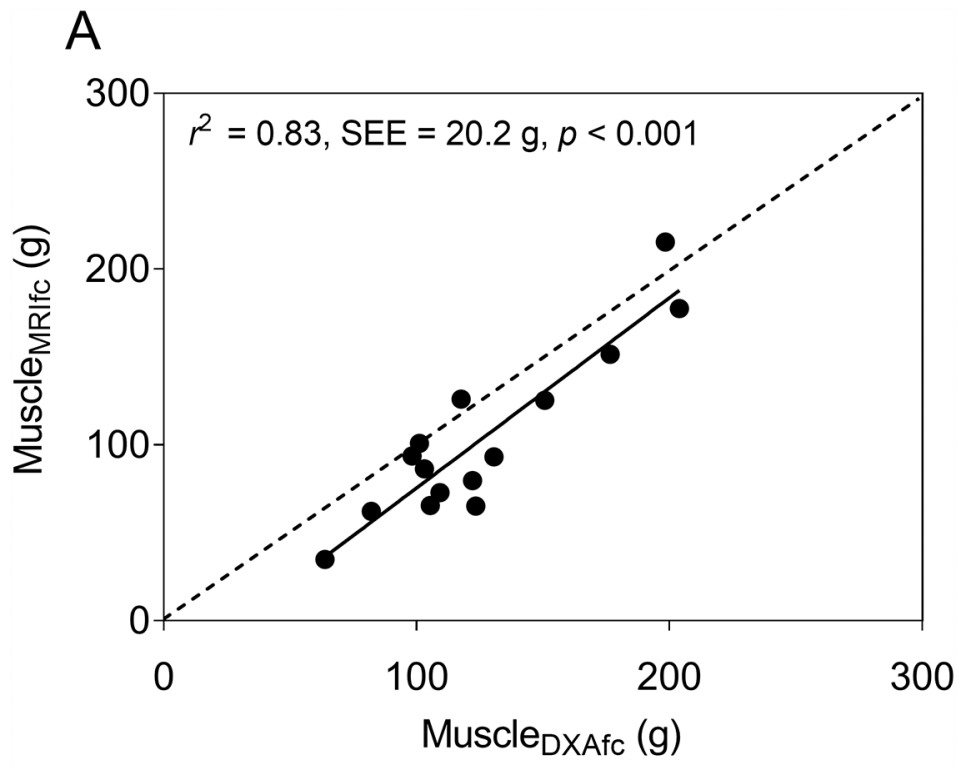

$\mathrm{B}$

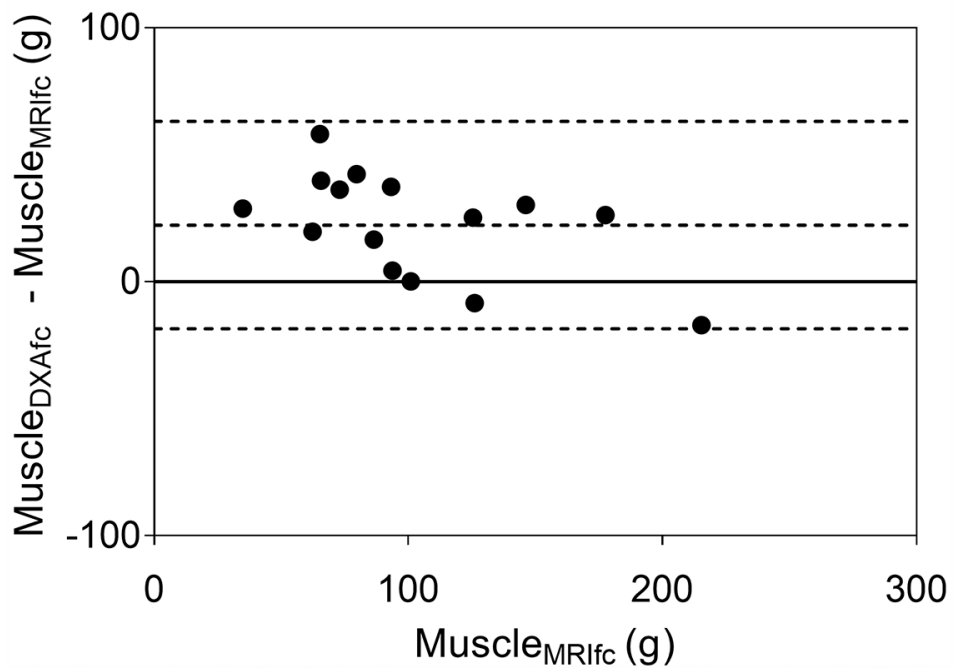

Fig. 4.

(A) The scatter plot shows midleg muscle mass adjusted for intramuscular fat concentration (Muscle $_{\text {MRIfc }}$ ) in children with cerebral palsy $(\mathrm{CP} ; \mathrm{n}=15)$ estimated using a dual-energy $\mathrm{X}$ ray absorptiometry (DXA) -based model (Muscle DXAfc $_{\text {) }}$ developed using data from typically developing children. Muscle ${ }_{\text {DXAfc }}$ was estimated using fat-free soft tissue mass (FFST) from DXA. The dotted line represents the line of identity. The solid line represents the regression line. (B) The Bland-Altman plot shows the level of agreement between Muscle DXAfc $_{\text {and }}$

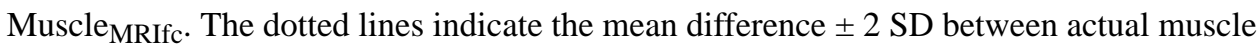
mass from MRI and the estimated muscle mass by the DXA-based model in children with CP. The solid line indicates no difference between actual and estimated muscle mass. 


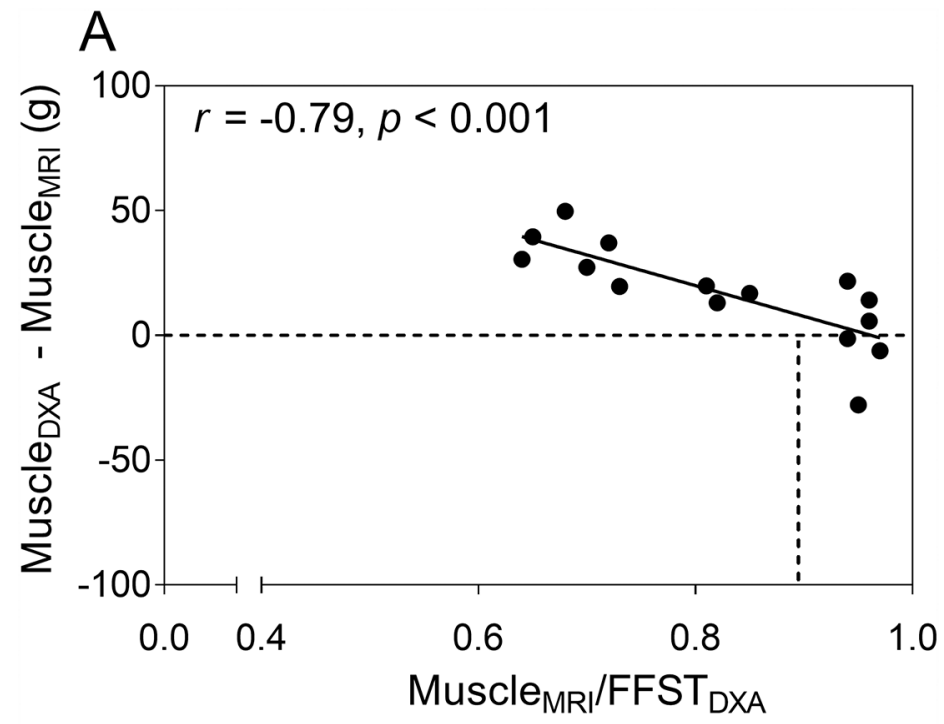

B

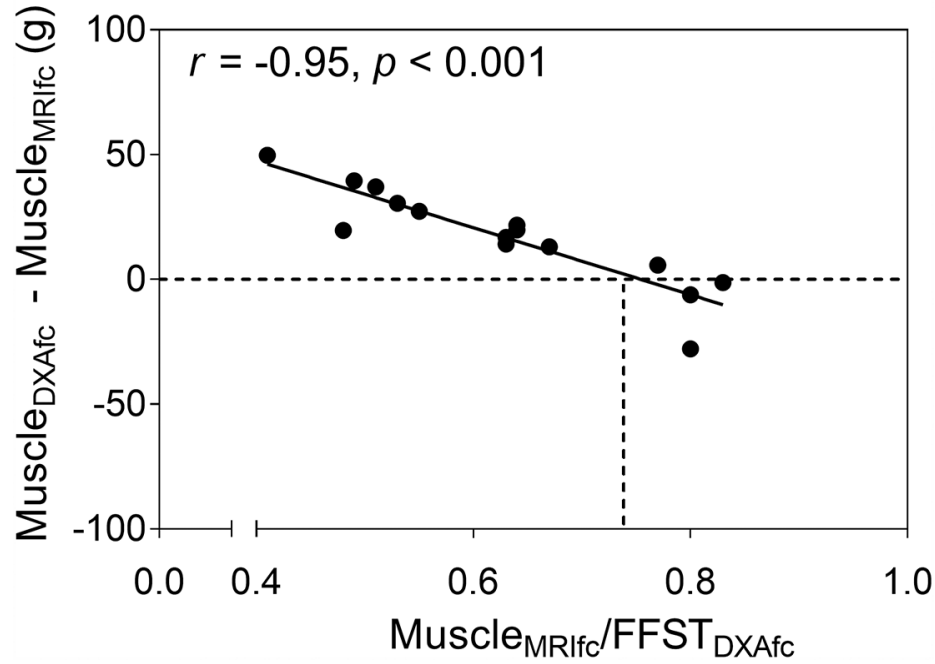

Fig. 5.

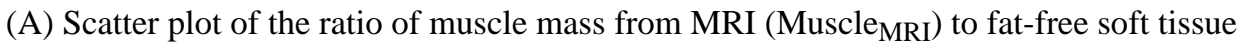

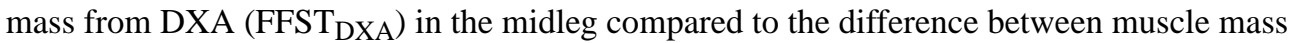
estimated by the DXA-based model using data from controls (Muscle DXA $_{\text {) and }}$ Muscle MRI $_{\text {I }}$ in children with cerebral palsy $(\mathrm{CP} ; \mathrm{n}=15)$. (B) Scatter plot of the ratio of muscle $\mathrm{MRI}_{\mathrm{I}}$ corrected for intramuscular fat concentration (Muscle MRIfc $_{\text {) }}$ to FFST $_{\text {DXA }}$ in the midleg compared to the difference between Muscle DXA $_{\text {corrected for intramuscular fat }}$

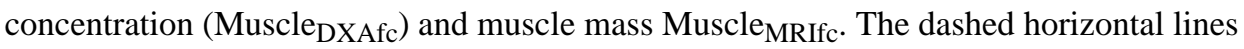
represent the points where the difference between muscle mass estimated from DXA and MRI are zero. The dashed vertical lines represent the mean ratios of muscle mass from MRI to $\operatorname{FFST}_{\text {DXA }}$ (0.88 for Muscle MRI $_{\text {FFST }}$ DXA $_{\text {and }} 0.75$ for Muscle $_{\text {MRIfc }} /$ FFST $_{\text {DXA }}$ ) for typically developing children. 


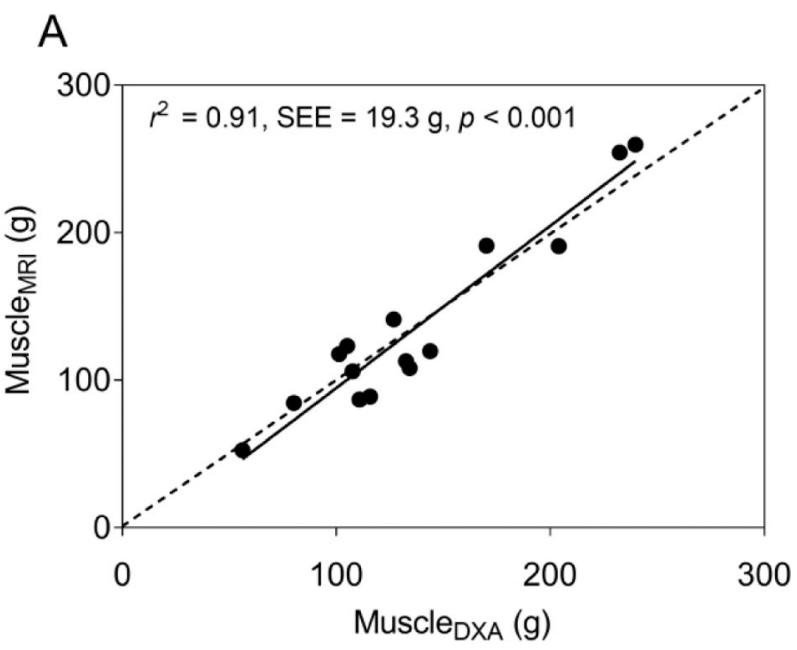

\section{B}

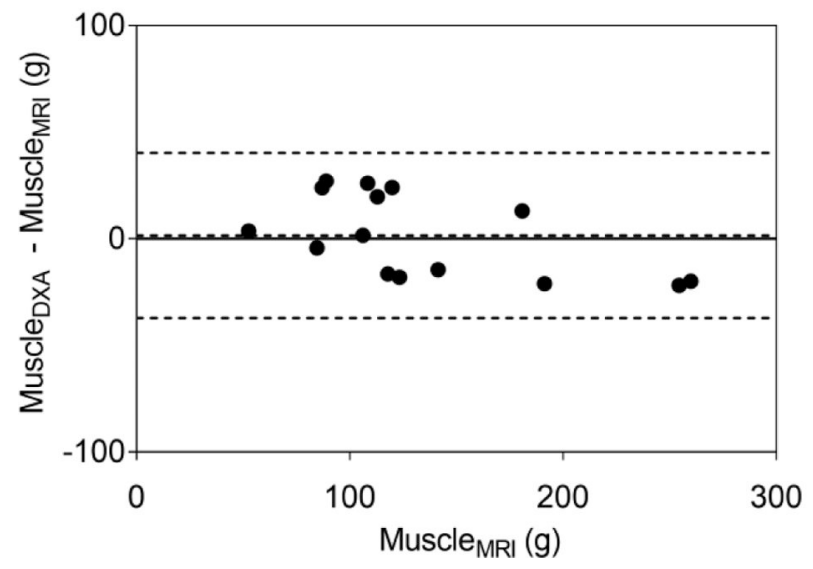

Fig. 6.

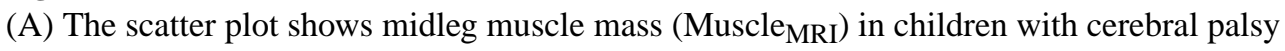
$(\mathrm{CP} ; \mathrm{n}=15$ ) estimated using a dual-energy X-ray absorptiometry (DXA) -based model (Muscle DXA $_{\text {) developed using data from children with CP. Muscle }}$ DXA was estimated using fat-free soft tissue mass (FFST) from DXA. The dotted line represents the line of identity. The solid line represents the regression line. (B) The Bland-Altman plot shows the level of ${\text { agreement between } \text { Muscle }_{\mathrm{DXA}} \text { and Muscle }}_{\mathrm{MRI}}$. The dotted lines indicate the mean difference \pm 2 SD between actual muscle mass from MRI and the estimated muscle mass by the DXA-based model. The solid line indicates no difference between actual and estimated muscle mass. 

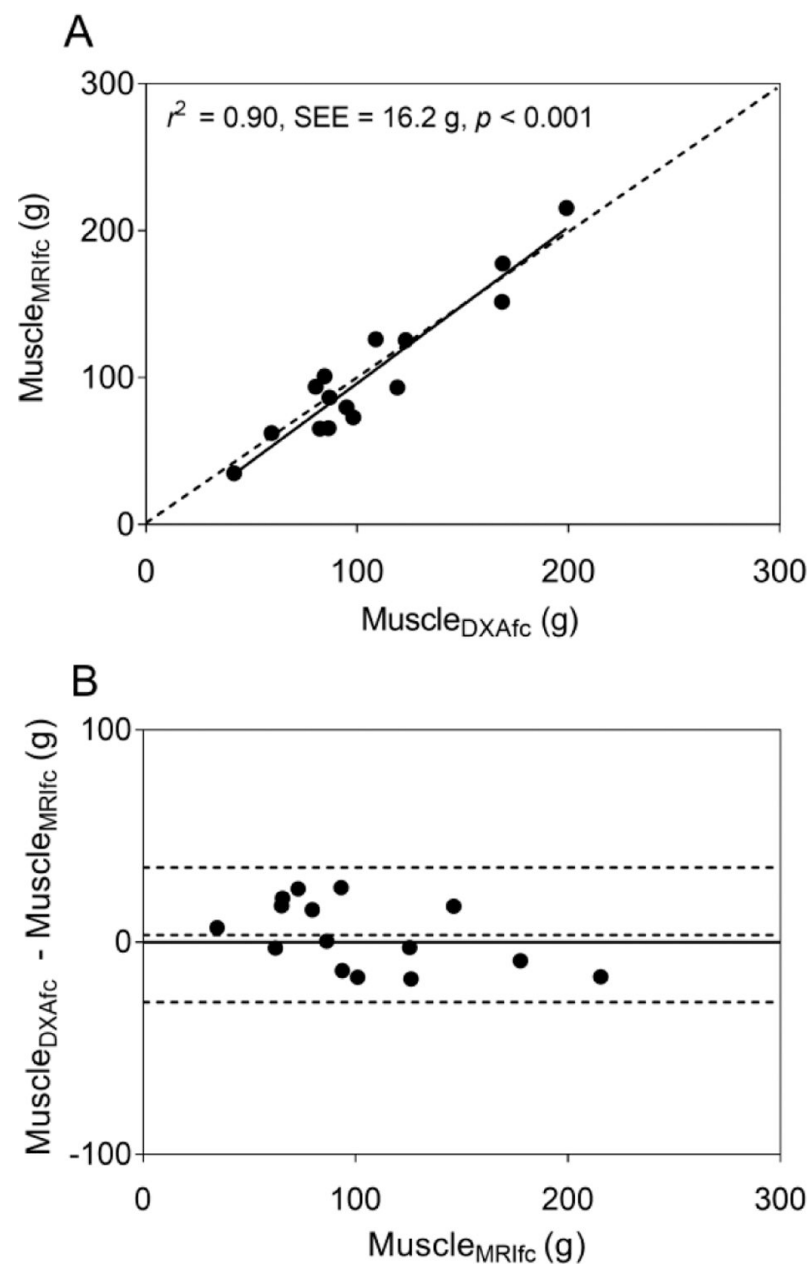

Fig. 7.

(A) The scatter plot shows midleg muscle mass adjusted for intramuscular fat concentration (Muscle $_{\text {MRIfc }}$ ) in children with cerebral palsy $(\mathrm{CP} ; \mathrm{n}=15)$ estimated using a dual-energy X-

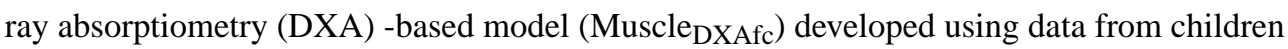

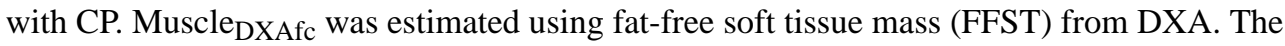
dotted line represents the line of identity. The solid line represents the regression line. (B) The Bland-Altman plot shows the level of agreement between Muscle DXAfc $_{\text {and }}$

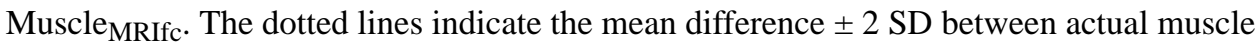
mass from MRI and the estimated muscle mass by the DXA-based model in children with $\mathrm{CP}$. The solid line indicates no difference between actual and estimated muscle mass. 
Table 1.

Physical characteristics, DXA measurements and MRI skeletal muscle mass in children with cerebral palsy (CP) and typically developing children (Con).

\begin{tabular}{lllcc}
\hline & CP $(\mathbf{n}=\mathbf{1 5})$ & Con $(\mathbf{n}=\mathbf{1 5})$ & $\boldsymbol{d}$ & $\boldsymbol{p}$ \\
\hline Ages (years) & $8.0 \pm 2.4$ & $8.3 \pm 2.1$ & 0.133 & 0.700 \\
Tanner stage (I/II/III) & & & & \\
$\quad$ Pubic hair & $11 / 3 / 1$ & $14 / 1 / 0$ & 0.550 & 0.345 \\
$\quad 11 / 4 / 0$ & $13 / 1 / 1$ & 0.137 & 0.595 \\
$\quad$ Testicular-penile/breast & $1.20 \pm 0.12$ & $1.30 \pm 0.11$ & 0.869 & 0.022 \\
Height (m) & $17 \pm 28$ & $58 \pm 30$ & 1.413 & $<0.001$ \\
Height (\%) & $24.7 \pm 9.3$ & $28.9 \pm 7.0$ & 0.510 & 0.067 \\
Body mass (kg) & $32 \pm 31$ & $60 \pm 26$ & 0.979 & 0.012 \\
Body mass (\%) & $16.9 \pm 3.4$ & $16.8 \pm 2.4$ & 0.034 & 0.461 \\
BMI (kg/m $)$ & $50 \pm 36$ & $55 \pm 28$ & 0.155 & 0.694 \\
BMI (\%) & $8 / 7$ & - & & \\
GMFCS (I/II) & $163 \pm 59$ & $263 \pm 77$ & 1.447 & $<0.001$ \\
FFST (g) & $125 \pm 78$ & $148 \pm 61$ & 0.321 & 0.161 \\
Fat mass (g) $_{\text {Muscle }}^{\text {MRI }(\mathrm{g})}$ & $136 \pm 61$ & $228 \pm 65$ & 1.485 & $<0.001$ \\
Intramuscular fat concentration $(\%)$ & $24 \pm 8$ & $15 \pm 3$ & 1.490 & 0.001 \\
Muscle $_{\text {MRIfc }}$ (g) & $103 \pm 48$ & $194 \pm 54$ & 1.786 & $<0.001$ \\
\hline
\end{tabular}

Values are mean \pm SD. BMI = body mass index. $\%$ for height, body mass and BMI reflects the percentile relative to age- and sex- based norms; $\%$ for intramuscular fat concentration reflects the percent fat within the muscle; $D X A=$ dual-energy X-ray absorptiometry; MRI = magnetic resonance imaging; GMFCS = gross motor function classification system; FFST = fat free soft tissue; MuscleMRI= muscle mass assessed by MRI;

MuscleMRIfc $=$ muscle mass assessed by MRI and corrected for fat concentration. $d=$ Cohen's $\mathrm{d}$. 
Table 2.

Statistical models developed to estimate midleg muscle mass MRI using data from typically developing children (Con) and children with cerebral palsy (CP).

\begin{tabular}{llcc}
\hline Group & Model & $\boldsymbol{R}^{2}$ & SEE (g) \\
\hline Con & 1. FFST $(\mathrm{g}) * 0.816+13.953$ & 0.96 & 14.1 \\
& 2. FFST $(\mathrm{g}) * 0.704+$ fat mass $(\mathrm{g}) * 0.221+10.924$ & 0.98 & 9.5 \\
$\mathrm{CP}$ & 3. FFST $(\mathrm{g}) * 0.986-24.577$ & 0.91 & 19.3 \\
\hline
\end{tabular}

All models are statistically significant, $p<0.001 ; \mathrm{MRI}=$ magnetic resonance imaging; FFST $=$ fat-free soft tissue; $\mathrm{n}=15 / \mathrm{group}$ 
Table 3.

Statistical models developed to estimate midleg muscle mass from MRI corrected for fat concentration using data from typically developing children (Con) and children with cerebral palsy (CP).

\begin{tabular}{llcc}
\hline Group & Model & $\boldsymbol{R}^{2}$ & SEE $(\mathrm{g})$ \\
\hline Con & 1. FFST $(\mathrm{g}) * 0.684+14.350$ & 0.96 & 10.7 \\
CP & 2. FFST $(\mathrm{g}) * 0.740-17.086$ & 0.83 & 20.9 \\
& 3. FFST $(\mathrm{g}) * 0.993$ - body mass $(\mathrm{kg}) * 2.123-5.766$ & 0.90 & 16.8 \\
\hline
\end{tabular}

All models are statistically significant, $p<0.001 ; \mathrm{MRI}=$ magnetic resonance imaging; FFST $=$ fat-free soft tissue; $\mathrm{n}=15 / \mathrm{group}$. 